\title{
Toward identifying reproducible brain signatures of obsessive-compulsive profiles: rationale and methods for a new global initiative
}

Helen Blair Simpson 1,2, Odile A. van den Heuvel ${ }^{3,4}$, Euripedes C. Miguel ${ }^{5,6}$, Y. C. Janardhan Reddy ${ }^{7}$, Dan J. Stein ${ }^{8}$, Roberto Lewis-Fernández ${ }^{1,2}$, Roseli Gedanke Shavitt ${ }^{5,6}$, Christine Lochner ${ }^{9}$, Petra J. W. Pouwels ${ }^{10}$,

Janardhanan C. Narayanawamy ${ }^{7}$, Ganesan Venkatasubramanian ${ }^{7}$, Dianne M. Hezel ${ }^{1,2^{*}}$ (D) Chris Vriend ${ }^{3,4}$, Marcelo C. Batistuzzo 5,6, Marcelo Q. Hoexter ${ }^{5,6}$, Niels T. de Joode ${ }^{3,4}$, Daniel Lucas Costa 5,6, Maria Alice de Mathis ${ }^{5,6}$, Karthik Sheshachala ${ }^{7}$, Madhuri Narayan $^{7}$, Anton J. L. M. van Balkom ${ }^{11,12}$, Neeltje M. Batelaan ${ }^{11,12}$,

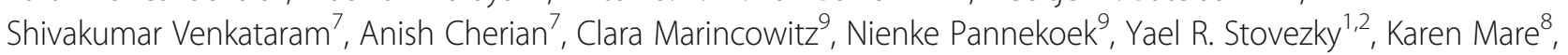
Feng Liu ${ }^{1,2}$, Maria Concepcion Garcia Otaduy ${ }^{5,6}$, Bruno Pastorello ${ }^{13}$, Rashmi Rao ${ }^{7}$, Martha Katechis ${ }^{1,2}$, Page Van Meter ${ }^{1,2}$ and Melanie Wall ${ }^{1,2}$

\begin{abstract}
Background: Obsessive-compulsive disorder (OCD) has a lifetime prevalence of 2-3\% and is a leading cause of global disability. Brain circuit abnormalities in individuals with OCD have been identified, but important knowledge gaps remain. The goal of the new global initiative described in this paper is to identify robust and reproducible brain signatures of measurable behaviors and clinical symptoms that are common in individuals with OCD. A global approach was chosen to accelerate discovery, to increase rigor and transparency, and to ensure generalizability of results.

Methods: We will study 250 medication-free adults with OCD, 100 unaffected adult siblings of individuals with OCD, and 250 healthy control subjects at five expert research sites across five countries (Brazil, India, Netherlands, South Africa, and the U.S.). All participants will receive clinical evaluation, neurocognitive assessment, and magnetic resonance imaging (MRI). The imaging will examine multiple brain circuits hypothesized to underlie OCD behaviors, focusing on morphometry (T1-weighted MRI), structural connectivity (Diffusion Tensor Imaging), and functional connectivity (resting-state $\mathrm{fMRI}$ ). In addition to analyzing each imaging modality separately, we will also use multi-modal fusion with machine learning statistical methods in an attempt to derive imaging signatures that distinguish individuals with OCD from unaffected siblings and healthy controls (Aim \#1). Then we will examine how these imaging signatures link to behavioral performance on neurocognitive tasks that probe these same circuits as well as to clinical profiles (Aim \#2). Finally, we will explore how specific environmental features (childhood trauma, socioeconomic status, and religiosity) moderate these brain-behavior associations.

(Continued on next page)
\end{abstract}

\footnotetext{
* Correspondence: dianne.hezel@nyspi.columbia.edu

Helen Blair Simpson, Odile A. van den Heuvel, Euripedes C. Miguel, Dan J.

Stein, Roberto Lewis-Fernández, Melanie Wall: Principal Investigators on grant

from the National Institutes of Mental Health (R01 MH113250).

${ }^{1}$ Columbia University Irving Medical Center, Columbia University, New York,

NY 10032, USA

${ }^{2}$ The New York State Psychiatric Institute, New York, NY 10032, USA

Full list of author information is available at the end of the article
}

(c) The Author(s). 2020 Open Access This article is distributed under the terms of the Creative Commons Attribution 4.0 International License (http://creativecommons.org/licenses/by/4.0/), which permits unrestricted use, distribution, and reproduction in any medium, provided you give appropriate credit to the original author(s) and the source, provide a link to the Creative Commons license, and indicate if changes were made. The Creative Commons Public Domain Dedication waiver (http://creativecommons.org/publicdomain/zero/1.0/) applies to the data made available in this article, unless otherwise stated. 
(Continued from previous page)

Discussion: Using harmonized methods for data collection and analysis, we will conduct the largest neurocognitive and multimodal-imaging study in medication-free subjects with OCD to date. By recruiting a large, ethno-culturally diverse sample, we will test whether there are robust biosignatures of core OCD features that transcend countries and cultures. If so, future studies can use these brain signatures to reveal trans-diagnostic disease dimensions, chart when these signatures arise during development, and identify treatments that target these circuit abnormalities directly. The long-term goal of this research is to change not only how we conceptualize OCD but also how we diagnose and treat it.

Keywords: Obsessive-compulsive disorder, Neuroimaging, fMRI, Unaffected siblings, Brain signatures, Neurocognitive, Global mental health

\section{Background}

Obsessive-compulsive disorder (OCD) has a lifetime prevalence of $2-3 \%[1,2]$ and results in reduced quality of life [3, 4], lower educational attainment [5], suicidality $[6,7]$, and even premature death $[8,9]$. A leading global cause of disability $[10,11]$, OCD contributes to significant economic burden [12] that is expected to increase over the next 20 years [13]. Even among individuals who receive evidence-based treatments [14], only some achieve remission $[15,16]$. One contributor to poor outcomes might be variation in neurobiological mechanisms that underlie different symptom profiles; addressing this variation with targeted treatments should improve care.

To begin to address this public health challenge, we launched an international collaboration with two specific aims. Our first aim is to identify reproducible brain signatures that distinguish individuals with OCD from unaffected siblings and healthy control subjects. Our second aim is to link these brain signatures to neurocognitive and clinical profiles observed in individuals with OCD. This approach is consonant with the United States (U.S.) National Institute of Mental Health's initiative on Research Domains Criteria ( $\mathrm{RDoC}$ ). The $\mathrm{RDoC}$ initiative seeks to develop a research classification system for psychopathology based on dimensions of neurobiology and observable behavior and to use these dimensions as targets for treatment development [17]. Our success could ultimately lead to the development of objective methods for diagnosing OCD and identifying new treatment targets for it, with relevance to diverse populations across the globe. Moreover, since some OCD symptom profiles overlap with those seen in anxiety disorders and other obsessive-compulsive-related disorders, the data generated by this study may pave the way for a transdiagnostic understanding of these brainbehavior associations and enable longitudinal studies that identify the point at which these brain signatures arise during development. This paper provides the rationale for our study design and describes our research methods.

\section{Rationale}

Why study $O C D$ ?

The clinical hallmarks of OCD are obsessions and compulsions. Obsessions include repetitive unwanted thoughts, images, impulses, or urges that typically generate distress; compulsions are repetitive behaviors or mental acts that the individual feels driven to perform [18]. In addition, up to $60 \%$ of individuals with OCD experience sensory phenomena, which are defined as subjective experiences that precede compulsions, and can include physical sensations, just-right sensations, and feelings of incompleteness [19-21]. Across countries and cultures, obsessions and compulsions cluster around common themes known as symptom dimensions. These dimensions include: concerns about contamination and cleaning compulsions; fear of harm and checking compulsions; need for symmetry/exactness and repeating, ordering, and counting compulsions; and forbidden or taboo thoughts (e.g., aggressive, sexual, religious obsessions) and related compulsions [22-24]. Because the core behaviors that characterize OCD-obsessions and compulsions-are relatively stereotyped across countries and cultures, focusing on this disorder offers an excellent test of the ability to use objective methods to identify reproducible brain circuit abnormalities that are linked to this discrete psychopathology.

Another reason to focus on OCD is that the imaging literature has identified a relatively consistent pattern of brain circuit abnormalities related to the disorder. Specifically, dysregulation of cortico-striatal-thalamocortical (CSTC) circuits is thought to underlie OCD symptoms [25, 26]. Neuroimaging studies [27] have identified structural and functional abnormalities in multiple nodes of these CSTC circuits, including the frontal cortices, the striatum, and the thalamus $[25,28]$. Abnormalities in limbic and fronto-parietal circuits have also been identified, and recent studies implicate the cerebellum [25, 26, 29-33]. These different circuits are depicted in Fig. 1, along with some of the key cognitive and behavioral processes that these circuits subserve [26]. 


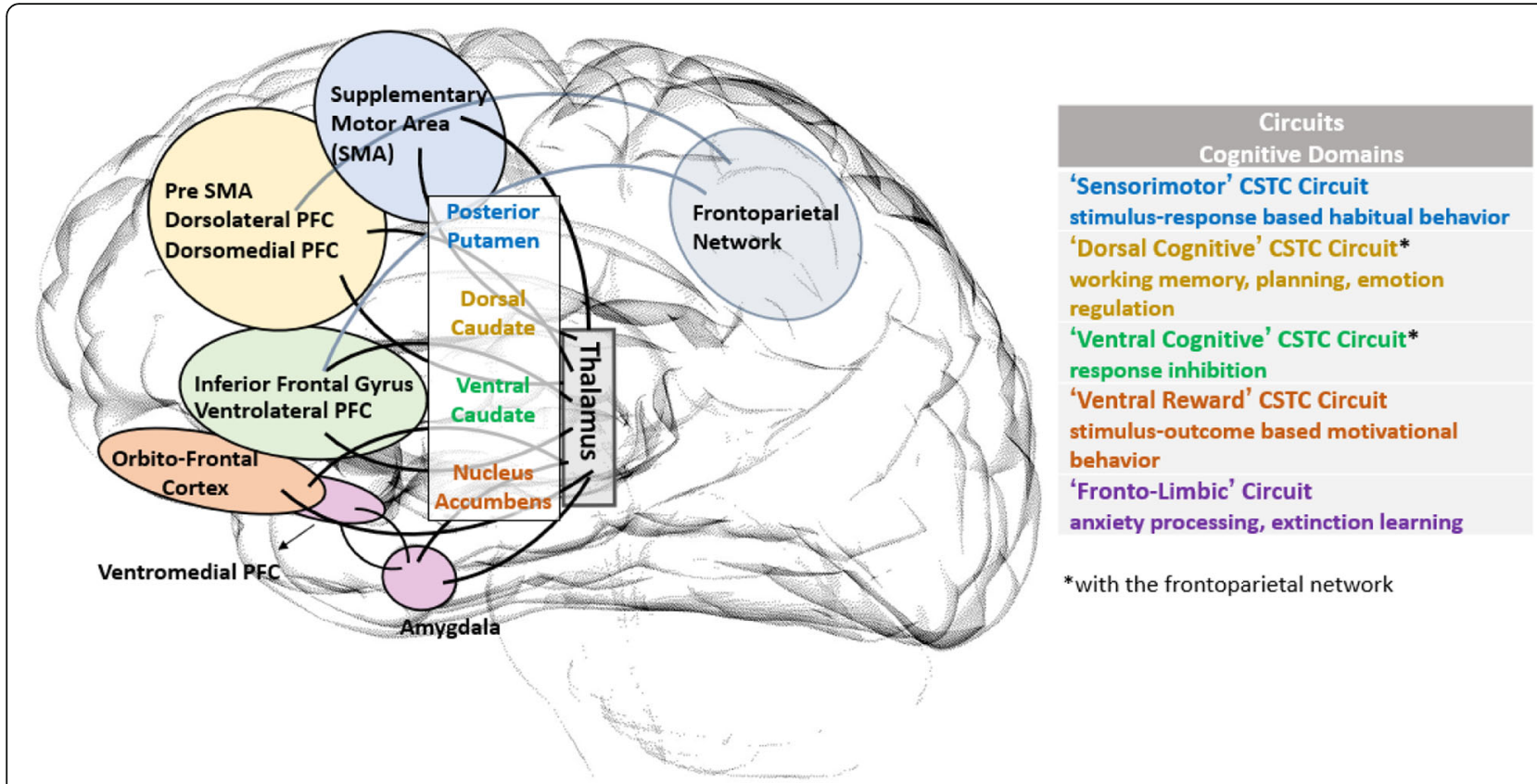

Fig. 1 Multiple Brain Circuits Contribute to OCD

However, prior imaging studies have important limitations that our study is designed to address. First, many have been single-site studies in small and historically Western, educated, industrialized, rich, and democratic (WEIRD) samples [27], and reproducibility of findings across sites has been variable. Meta- and mega-analyses (like those done by the OCD Brain Imaging Consortium (OBIC) and the initiative for Enhancing Neuroimaging and Genetics through Meta-analyses (ENIGMA) [3437]) have been conducted, resulting in very large samples. These analyses pool existing data from multiple sites that use different inclusion criteria, clinical measures, and imaging methods. This variation not only introduces potential confounds, but also precludes linking brain findings to detailed cognitive or clinical profiles because of the lack of harmonization of such measures.

Second, most large-scale studies have been anatomical [33], examining brain volume only. Few large-scale studies have used diffusion weighted imaging (DWI) to assess structural connectivity [38-43] or resting state functional MRI (rs-fMRI) to assess functional connectivity [41, 44-54]. Moreover, existing DWI and rs-fMRI studies in OCD have used different acquisition and/or analytic methods, making lack of replication hard to interpret. In addition, many OCD subjects were on psychotropic medication at the time of imaging, despite the known effects on morphometry [37], DWI [55, 56], and rs-fMRI measures [47, 57].

Third, few studies have analyzed these imaging modalities in combination, despite the increasing recognition that multi-modal analysis of imaging data can help identify brain-behavior links [58]. Finally, although some single-site studies report significant correlations between brain circuit abnormalities (using DWI and rs-fMRI) and different neurocognitive [43] and clinical profiles $[47,59]$, the reproducibility of these findings needs rigorous testing across larger and more diverse populations using harmonized methods not only for brain imaging, but also for clinical phenotyping and neurocognitive testing [60].

To address these limitations, we will recruit 250 medication-free individuals with OCD, 100 unaffected siblings of individuals with OCD, and 250 healthy control subjects $(\mathrm{HCs})$ at five expert research sites that span five countries (Brazil, India, Netherlands, South Africa, U.S.; see Fig. 2). Using imaging methods chosen explicitly because of their potential adaptation for clinical use, we will examine multiple brain circuits thought to underlie OCD behaviors, focusing on morphometry (T1weighted MRI), structural connectivity (DWI), and functional connectivity (rs-fMRI).

Our first aim is to identify imaging signatures that distinguish individuals with OCD from unaffected siblings and HCs; we will accomplish this by analyzing each modality with standardized protocols and by using multimodal fusion with modern machine learning statistical methods. We hypothesize that individuals with OCD will show altered structure and function within specific frontal-striatal, frontal-limbic, and frontal-parietal circuits. Our second aim is to then link these imaging signatures both to behavioral performance on cognitive tasks that probe these same circuits and to discrete 


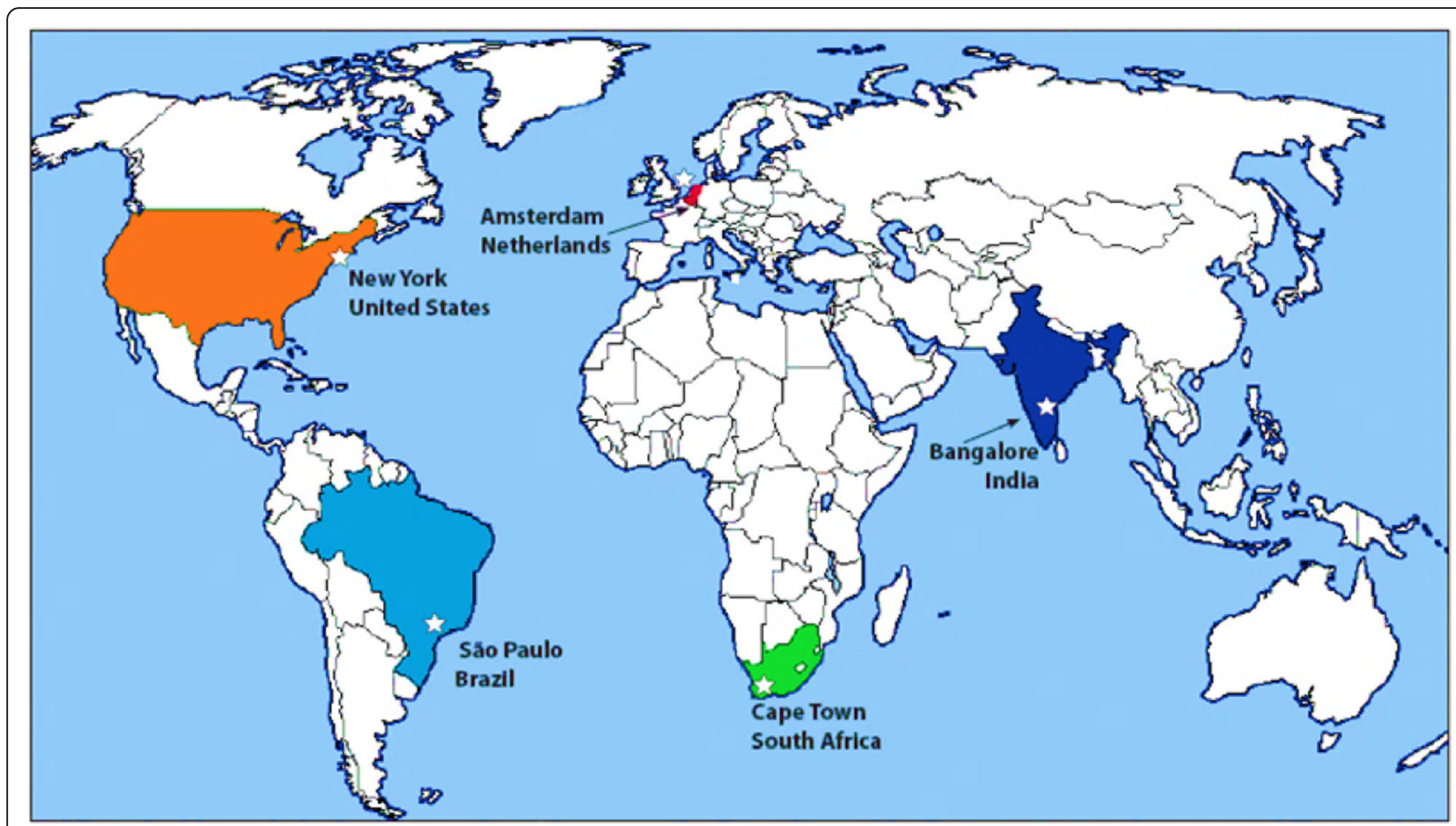

Fig. 2 The Five Collaborating Sites

clinical profiles. We hypothesize that imaging signatures capturing different circuit abnormalities will correlate with behavioral performance on cognitive tasks that probe these same circuits as well as with discrete clinical profiles. Given the ethno-socio-cultural diversity of our sample, we will also explore how specific environmental features (childhood trauma, socioeconomic status, and religiosity) may moderate these brain-behavior links.

\section{Why take a global approach?}

Although OCD and its core features are observed across the globe, the specific presentation of OCD varies among individuals. Specifically, patients differ from one another both in the specific content of their OCD symptoms and in many other clinical features, including symptom severity, age of onset, course of illness, degree of insight, comorbidity, family history, and degree of functional impairment [61-64]. Some have reported that this variation in clinical presentation is reflected in different brain abnormalities [10, 27, 62, 65-68]. However, other differences in disease expression may be better explained by specific environmental and cultural factors. Therefore, recruiting a large, ethno-culturally diverse sample will enable us to test whether there are robust biosignatures of specific OCD clinical profiles that transcend countries and cultures and that could be used as targets for new treatments with relevance to populations across the globe.
Our sites were chosen for multiple reasons. First, all have expertise in OCD as well as the necessary clinical and imaging research infrastructure. Second, all can recruit an OCD sample that is similar in core OCD features and educational levels, yet diverse in socio-cultural features, enabling us to test how robust and replicable our imaging signatures are. Third, all can recruit medication-free OCD subjects in a timely and economical way, enabling us to collect this large sample within the funding boundaries of a single R01 from NIMH. Fourth, each is a center of excellence for research, training, and treatment of OCD in its respective country. Thus, our findings will have both local and global impact.

Finally, these sites had a successful track record of working together. For example, the Principal Investigators have worked together to revise the guidelines for the World Health Organization for OCD and Related Disorders [62] and have also collaborated in the ENIGMA-OCD consortium [69]. By joining forces for this study, we seek to shift the research model from local to global expertise, increase rigor and transparency, and accelerate discovery by developing a circuit-based approach to cognitive and clinical dimensions.

\section{Why include siblings?}

Given the high heritability of OCD [28], we will also include in our sample individuals who have a biological sibling with OCD but do not have OCD themselves 
("unaffected" siblings). This approach has been used successfully in several prior imaging and neurocognitive studies in OCD [41, 54, 70-74]. Including unaffected siblings will support and strengthen our primary aims by allowing us to identify: (1) brain abnormalities that are present in OCD patients but not in their unaffected siblings or $\mathrm{HC}$ subjects (helping to define brain signatures that are most strongly linked to the disease state); (2) brain abnormalities that OCD patients and unaffected siblings share compared to matched HCs (helping to define brain signatures that may be linked to disease vulnerability); and (3) brain differences that are found in unaffected siblings but in neither HCs nor OCD patients (providing possible clues about resilience or compensatory brain mechanisms in unaffected siblings).

\section{Why include environmental variables in a brain imaging study?}

Some differences in OCD disease expression are hypothesized to be driven more by the local environment or contextual factors (e.g., relative severity of specific symptom dimensions, specific religious beliefs, degree of impairment/quality of life) than biology per se [75-78]. However, these factors have neither been examined in a large global sample, nor correlated with imaging and neurocognitive profiles. To begin to address this gap, we will use clinical assessments to explore disease expression across the sites. In addition, we will examine three specific environmental factors- childhood trauma, socioeconomic status (SES), and religiosity - to determine if they moderate the link between our neuroimaging signatures and clinical and cognitive profiles. We will focus on childhood trauma and SES because they have been identified as environmental risk factors for OCD [7981]. Moreover, they have known effects on brain structure in healthy people [82-84], the potential to confound imaging data [83, 85-90], and have been positively associated with larger right orbitofrontal cortex volume in individuals with OCD [91]. Religiosity (defined as the salience of religious experience in a person's life) has been associated with higher OCD symptoms, regardless of religion, and will thus also be explored within this multicultural study context. Finally, we will capitalize on this large and international sample to collect qualitative data with the Cultural Formulation Interview (CFI) [92] to explore the role of culture in the presentation and understanding of illness in those with OCD.

\section{Methods}

\section{Study design}

This project is an observational study that includes brain imaging and clinical and neurocognitive assessments, as described below.

\section{Setting(s)}

This study is being conducted at five expert OCD research sites, including: the Obsessive-Compulsive Spectrum Disorders Program led by Drs. Euripedes Miguel and Roseli Shavitt at the Institute and Department of Psychiatry, Hospital das Clinicas-HCFMUSP, University of São Paulo Medical School in São Paulo, Brazil; the OCD program led by Dr. Janardhan Reddy at the National Institute of Mental Health and Neurosciences in Bangalore, India; the Neuropsychiatry section led by Dr. Odile van den Heuvel at the Department of Psychiatry/Anatomy \& Neurosciences, Amsterdam University Medical Centers (location Free University (VU) Medical Center) in collaboration with the Anxiety and OCD Program of Mental Health Institute GGZ inGeest, Amsterdam, the Netherlands; the OCD program led by Drs. Dan Stein and Christine Lochner at the South African Medical Research Council Unit on Risk and Resilience in Mental Disorders in Cape Town, South Africa; and the Center for OCD and Related Disorders led by Dr. Helen Blair Simpson at the New York State Psychiatric Institute/ Columbia University, New York, NY, USA. All sites have experience conducting OCD research as well as the necessary clinical research and imaging infrastructure (e.g., 3.0 T MRI machines).

This project uses a collaborative leadership model, with Dr. Simpson as the contact PI for NIMH. The executive committee is comprised of the principal investigators (PIs) at each site and their key personnel; each PI is responsible for the study at his or her site, and the committee meets by videoconference twice per month. Each site will recruit the same number of subjects and use harmonized methods for clinical assessment, neurocognitive testing, and imaging acquisition. The institutional review board or ethics board at each site (named above) has reviewed and approved the study procedures. All subjects will provide written informed consent prior to participation.

\section{Subjects}

A total of 250 medication-free OCD patients, 100 unaffected siblings, and 250 healthy control subjects will be recruited across all five sites (50 OCD patients, 50 healthy control subjects, and 20 unaffected siblings per site). The OCD and healthy control samples will be matched on age, gender, and educational level (within and between sites). Inclusion and exclusion criteria are outlined in Table 1 . OCD subjects must have OCD as their principal diagnosis with at least moderate severity. Unaffected siblings must have a first-degree sibling with OCD but not meet criteria for OCD themselves. 
Table 1 Inclusion and Exclusion Criteria

\begin{tabular}{|c|c|c|c|}
\hline & OCD $(n=250)$ & Unaffected Siblings $(n=100)$ & Healthy Controls $(n=250)$ \\
\hline $\begin{array}{l}\text { Inclusion } \\
\text { Criteria }\end{array}$ & $\begin{array}{l}\text { - } 18-50 \text { years old } \\
\text { - Principal diagnosis of OCD } \\
\text { - YBOCS } \geq 16\end{array}$ & $\begin{array}{l}\cdot 18-50 \text { years old } \\
\cdot \text { Has sibling with OCD }\end{array}$ & . $18-50$ years old \\
\hline \multirow[t]{2}{*}{$\begin{array}{l}\text { Exclusion } \\
\text { Criteria }\end{array}$} & $\begin{array}{l}\text { - Lifetime diagnosis of psychosis, bipolar } \\
\text { disorder, anorexia, autism, or Tourette disorder } \\
\text { - Current chronic tic disorder, substance-use dis- } \\
\text { order, binge-eating disorder, bulimia, or } \\
\text { suicidality } \\
\text { - Current use of psychotropic medications or } \\
\text { CBT for OCD }\end{array}$ & $\begin{array}{l}\text { - Current or lifetime psychiatric } \\
\text { disorder other than MDD or anxiety } \\
\text { disorders } \\
\text { - Current use of psychotropic } \\
\text { medications }\end{array}$ & $\begin{array}{l}\text { - Current or lifetime psychiatric disorder other } \\
\text { than MDD or anxiety disorders (if not in past } \\
\text { year) } \\
\text { - Current or past use of psychotropic } \\
\text { medications } \\
\text { - First-degree relative with OCD or tic disorder }\end{array}$ \\
\hline & $\begin{array}{l}\text { - Major medical or neurological diseases } \\
\text { - IQ }<80 \\
\text { - Contraindications to MRI }\end{array}$ & & \\
\hline
\end{tabular}

OCD Obsessive-compulsive disorder, YBOCS Yale-Brown Obsessive-Compulsive Severity Scale, CBT Cognitive-behavioral therapy, IQ Intelligence quotient, MRI Magnetic resonance imaging, MDD Major depressive disorder

\section{Measures}

\section{Screening for eligibility}

To determine eligibility, a trained rater will conduct a clinical evaluation with the Structured Clinical Interview for DSM-5 (SCID) to confirm diagnosis, the Yale-Brown Obsessive-Compulsive Scale (Y-BOCS) [93, 94] to assess OCD severity, and an assessment of IQ. Screening will also include questions about treatment history, medical history, family psychiatric history, and tic disorder. Those eligible and interested will be enrolled after providing written informed consent.

Standardizing assessment of IQ presented a significant challenge given that no single IQ measure has been validated across all five countries and languages. Consequently, each site selected a measure of IQ that has been validated in the appropriate languages for its respective country and can yield a general IQ score as well as an estimate of performance and verbal domains (Brazil: Wechsler Abbreviated Scale of Intelligence First Edition (WASI-I) [95]; India: Binet Kamat Test [96]; Netherlands: selected subscales from the Wechsler Adult Intelligence Scale Fourth Edition (WAIS-IV) [97]; South Africa: Wechsler Abbreviated Scale of Intelligence Second Edition (WASI-II) [98]; USA: WASI-II). The IQ test will be administered by trained raters at each site.

\section{Clinical evaluations}

A standardized protocol will be used at all sites to clinically assess subjects in their respective language (i.e., Afrikaans, Dutch, English, Kannada, or Portuguese). This protocol will include the Common Data Elements required by NIMH as well as validated clinical measures that have been used around the globe and that tap different clinical profiles common in individuals with OCD. In addition, validated measures of trauma history, SES, and religiosity will also be used. These measures are shown in Table 2 and include semi-structured interviews performed by a trained rater and self-report questionnaires. We will also collect qualitative data using the Cultural Formulation Interview (CFI) [92] to explore the role of culture in the presentation and understanding of illness in those with OCD.

\section{Neurocognitive assessment}

Trained experimenters at each site will assess subjects using a computerized neurocognitive protocol. Tasks were chosen that are: 1) valid (i.e., known to probe brain circuits and domains of cognitive dysfunction that are implicated in OCD (see Fig. 1); 2) generalizable (i.e., in the public domain and with minimal reliance on language); 3) reproducible (i.e., computerized and standardized); and 4) consonant with the NIMH's RDoC matrix [113]. The brain circuits and domains of cognitive function that these tasks will probe are shown in Table 3. All tasks will be completed outside of the scanner.

Although imaging studies in healthy subjects have shown that these tasks probe the brain circuits implicated in OCD, prior OCD studies have revealed mixed behavioral effects with these tasks, with only some finding behavioral deficits in individuals with OCD relative to healthy control subjects [129-131]. There are many potential reasons for these mixed results, including the fact that studies used different task versions and many tested OCD subjects who were taking medication and/or had different types of comorbid conditions. Moreover, not all prior studies measured and controlled for IQ [132]. Finally, if the behavioral deficits are subtle, they will be more difficult to detect consistently across samples, especially if samples are small. In the current study, we attempt to address these limitations by using computerized and standardized task versions, only testing subjects who are medication-free, measuring IQ, and recruiting a large sample. 
Table 2 Clinical Domains and Measures

\begin{tabular}{|c|c|}
\hline Clinical Domains & Measures \\
\hline \multicolumn{2}{|c|}{ Obsessive-Compulsive Profiles } \\
\hline Total Severity & Yale-Brown Obsessive-Compulsive Scale (Y-BOCS) [93, 94]; Obsessive-Compulsive Inventory-R ${ }^{a}[99]$ \\
\hline $\begin{array}{l}\text { Dimension } \\
\text { Severity }\end{array}$ & Dimensional Yale-Brown Obsessive-Compulsive Scale (DY-BOCS) [100] \\
\hline Insight & Brown Assessment of Beliefs Scale (BABS) [101] \\
\hline $\begin{array}{l}\text { Sensory } \\
\text { Phenomena }\end{array}$ & University of São Paulo Sensory Phenomena Scale (USP-SPS) [102] \\
\hline Age of Onset & Structured Clinical Interview for DSM-5 (SCID) [103]; Center for OCD and Related Disorders Age of Onset and Course Form \\
\hline Depression & Hamilton Depression Rating Scale (HAM-D) [104] \\
\hline Anxiety & Hamilton Anxiety Rating Scale (HAM-A) [105] \\
\hline $\begin{array}{l}\text { Other Clinical } \\
\text { Profiles }\end{array}$ & $\begin{array}{l}\text { Autism Spectrum Quotient }{ }^{\mathrm{a}} \text { [106]; Center for OCD and Related Disorders Tic Questionnaire; Disgust Propensity and Sensitivity } \\
\text { Scale }^{\mathrm{a}} \text { [107]; Impulsive-Compulsive Behaviours Checklist }{ }^{\mathrm{a}} \text { [108]; Obsessive-Compulsive Personality Disorder Questionnaire }\end{array}$ \\
\hline Functioning & World Health Organization Disability Assessment Schedule 2.0 (WHODAS) [109] \\
\hline \multicolumn{2}{|l|}{ Environmental } \\
\hline $\begin{array}{l}\text { Socioeconomic } \\
\text { Status }\end{array}$ & Work and Meaning Inventory (WAMI) ${ }^{\mathrm{a}}[110]$; \\
\hline Trauma & Childhood Trauma Questionnaire ${ }^{a}[111]$ \\
\hline Religiosity & Religious Behaviors and Beliefs Questionnaire ${ }^{a}$ [112]; \\
\hline
\end{tabular}

${ }^{a}$ Self-report measure; all other measures are administered by a trained clinician

\section{MRI imaging}

All subjects will undergo multi-modal MRI in a 3.0 Tesla whole-body scanner equipped with a 32-channel (or 48-channel at the U.S. site) phased-array head coil. Details about the MRI scanners and sequences are provided in Table 4. Sequences include: 1) highresolution $3 \mathrm{D}$ T1 weighted structural imaging using MPRAGE according to the ADNI3 protocol with 1 $\mathrm{mm}$ isotropic resolution; 2) multi-shell diffusion weighted imaging (DWI), and 3) resting-state fMRI (10 min, eyes closed). In addition, we added a highresolution 3D phase-sensitive inversion recovery
(PSIR) sequence to optimize segmentation of deep grey matter structures. All image processing will be conducted by the Netherlands site using processing scripts developed by the Netherlands site and ENIGMA [69].

We chose standard imaging sequences for structural and functional imaging, which are commercially available or feasible to implement on most clinical scanners. Our rationale was that overly specialized paradigms that can be conducted only in a few WEIRD [27] laboratories will have limited clinical impact across most of the world. In contrast, if we

Table 3 Cognitive Tasks and Domains

\begin{tabular}{|c|c|c|c|}
\hline Brain Circuit & Cognitive Domain & Cognitive Task & Outcomes \\
\hline \multirow{2}{*}{$\begin{array}{l}\text { Dorsal } \\
\text { "cognitive" } \\
\text { CSTC }^{a}\end{array}$} & Working Memory & Visual Spatial N-Back [71] & Percent of correct trials overall and per condition \\
\hline & Planning & Tower of London $[114,115]$ & Percent of correct trials overall and per task load \\
\hline $\begin{array}{l}\text { Ventral } \\
\text { "cognitive" } \\
\text { CSTC }^{a}\end{array}$ & Response Inhibition & Stop-Signal $[70,116,117]$ & Stop signal reaction time \\
\hline $\begin{array}{l}\text { Ventral } \\
\text { "reward" CSTC }\end{array}$ & Reward Processing & $\begin{array}{l}\text { Temporal Discounting }[118- \\
\text { 121] }\end{array}$ & $\begin{array}{l}\text { Discount rate parameter on the intertemporal choice task [and risk } \\
\text { aversion parameter on the risk aversion task] }\end{array}$ \\
\hline Frontal-Limbic & $\begin{array}{l}\text { Emotion Regulation / } \\
\text { Executive Control }\end{array}$ & Emotional Stroop $[115,122]$ & Mean reaction time and Stroop effect \\
\hline $\begin{array}{l}\text { Sensorimotor } \\
\text { CSTC }\end{array}$ & Motor Learning & $\begin{array}{l}\text { Motor Sequencing }[123- \\
126]\end{array}$ & Learning rate, speed and accuracy, variability in motor performance \\
\hline $\begin{array}{l}\text { Combination of } \\
\text { CSTC }\end{array}$ & $\begin{array}{l}\text { Reward learning / } \\
\text { Decision Making }\end{array}$ & $\begin{array}{l}\text { Two Stage Reinforcement } \\
\text { Learning }[127,128]\end{array}$ & $\begin{array}{l}\text { Proportion of decisions to repeat a rewarded choice vs. an unrewarded } \\
\text { choice following either a common or rare transition }\end{array}$ \\
\hline
\end{tabular}

${ }^{a}$ With the frontoparietal network 
Table 4 MRI Parameters

\begin{tabular}{|c|c|}
\hline \multicolumn{2}{|l|}{ MRI Scanner } \\
\hline Brazil & Philips Achieva 3.0 T \\
\hline India & Philips Ingenia 3.0 T CX \\
\hline Netherlands & GE 3.0 T Discovery MR750 \\
\hline $\begin{array}{l}\text { South } \\
\text { Africa }\end{array}$ & Siemens MAGNETOM Skyra 3.0T \\
\hline U.S. & GE 3.0 T SIGNA Premier \\
\hline Head coil & 32-channel or 48-channel ${ }^{\#}$ \\
\hline \multicolumn{2}{|l|}{ MRI Sequences } \\
\hline $\begin{array}{l}\text { Structural } \\
\mathrm{T} 1\end{array}$ & $\begin{array}{l}\text { 3D sagittal T1-weighted MP-RAGE according to ADNI-3 } \\
\text { protocol }(1 \times 1 \times 1 \mathrm{~mm} \text { resolution) }\end{array}$ \\
\hline rs-fMRI & $\begin{array}{l}\text { T2*-weighted echo-planar images while subjects are } \\
\text { awake and keep their eyes closed ( } 10 \mathrm{~min}, \mathrm{TR}=2200 \mathrm{~ms} \text {, } \\
\mathrm{TE}=28 \mathrm{~ms}, 3.3 \times 3.3 \mathrm{~mm} \text { in-plane resolution, } 3 \mathrm{~mm} \text { slices } \\
\text { with } 0.3 \mathrm{~mm} \text { gap) }\end{array}$ \\
\hline DWI & $\begin{array}{l}\text { multi-shell single spin echo DWI ( } 80 \text { interleaved } \\
\text { volumes: } 7 \text { b0, } 25 \text { b1000, } 24 \text { b2000, } 24 \text { b3000, } 2.5 \times \\
2.5 \times 2.5 \mathrm{~mm} \text { ) }\end{array}$ \\
\hline PSIR & $\begin{array}{l}\text { T1-weighted image with improved SNR and gray-white } \\
\text { matter contrast }(1 \times 1 \times 1 \mathrm{~mm} \text { resolution) }\end{array}$ \\
\hline
\end{tabular}

All sites use a 32-channel head coil, except NYC which uses a 48-channel head coil. Abbreviations: $r s-f M R I$ Resting-state functional Magnetic Resonance Imaging, DWI Diffusion weighted Imaging, PSIR Phase sensitive inversion recovery

identify robust biosignatures of psychopathology across countries/cultures using standardized imaging, our findings can then be leveraged by many.

\section{Study flow}

The timing of the assessments and the flow of subjects through the study are outlined in Fig. 3; the specific measures used for each clinical domain are listed in Table 2. The clinical assessments, neurocognitive testing, and MRI scanning will be conducted within 7 days (and ideally within $48 \mathrm{~h}$ ) to be able to correlate these measures.

\section{Study status}

The study is ongoing and currently all sites are collecting data at the time of this paper's submission.

\section{Data analytic plan and power analysis}

We will collect data from a total of 600 individuals (250 OCD, 100 unaffected siblings, and $250 \mathrm{HC}$ ) across five sites. Our first aim is to identify reproducible neuroimaging signatures that distinguish individuals with $O C D$ from unaffected siblings and healthy controls. To accomplish this aim, each imaging modality (anatomical, DWI, and rs-fMRI) will be analyzed using standardized protocols for uni-modal analyses; fusion of multi-modal imaging measures using modern machine learning or multilayer analyses $[58,133]$ will also be used to identify data-driven signatures that distinguish the groups. Our second aim is to link these neuroimaging signatures to behavioral performance on the cognitive tasks that probe these same circuits (Table 4) and to different clinical profiles commonly seen in individuals with OCD (e.g., different symptom dimensions, degree of insight, age of onset, comorbidity). We will also explore whether three environmental factors (childhood trauma, social economic status, and religiosity) moderate the link between our neuroimaging signatures and our OCD clinical and cognitive profiles.

With 250 medication-free OCD subjects, 250 demographically matched HCs, and 100 unaffected siblings, we will have $80 \%$ power while controlling for Type 1 error to $5 \%$ to detect effect size differences (i.e., group differences on a standardized scale) of Cohen's $\mathrm{d}=0.25$ between OCD and HC groups, and $\mathrm{d}=0.33$ between unaffected siblings and either OCD or HC subjects. We note that even within sites (i.e., within each country) our sample size provides $>80 \%$ power to detect effect sizes of $d=0.57$ between $\mathrm{OCD}$ and $\mathrm{HC}$ subjects and $\mathrm{d}=0.80$ when comparing either group to unaffected siblings. We will have $80 \%$ statistical power to test correlations of sizes as small as $r=0.12$ across all subjects at all sites $(r=0.25$ within site $)$

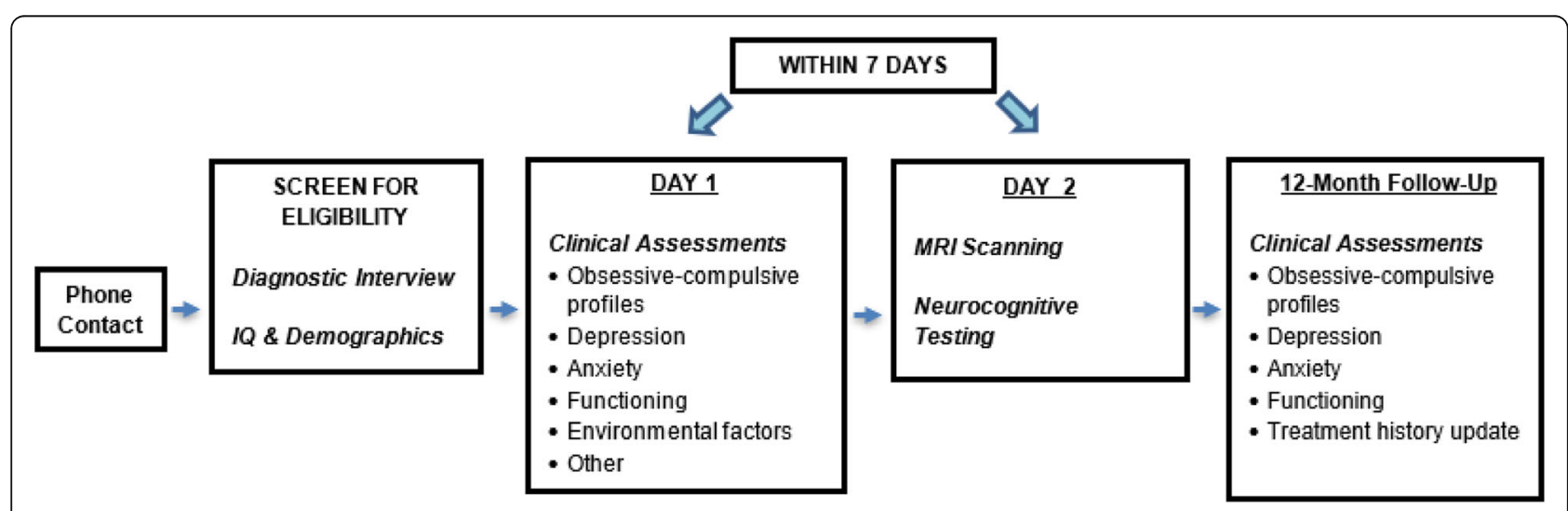

Fig. 3 Study Design 
and $r=0.18$ across all OCD subjects at all sites $(r=0.40$ within site). Previous studies, including our own, have found moderate to large effect sizes (i.e., $\mathrm{d}=0.35-0.90$ ) in neuroimaging measures corresponding to our OCD versus $\mathrm{HC}$ hypotheses, and recent studies have found strong correlations (e.g., 0.40 or greater) between neuroimaging measures and cognitive and clinical profiles [43, 47, 59]. Hence, the current study is powered to identify previously found or smaller effect sizes across all sites. Moreover, we expect statistical power for detecting OCD neuroimaging profiles to be increased through our fusion of multi-modal imaging data with machine learning statistical methods, which optimally combine all information on the same individual.

\section{Quality control Overview}

Each site will be responsible for all research procedures at its setting and will contribute to the cross-site workgroups as described below. The U.S. site is responsible to NIMH for the overall conduct of the study and will be directing the data management and statistical plan. The Netherlands site will oversee all imaging procedures, including harmonization of the imaging sequences, review of image quality, and image processing.

To ensure quality control and cross-site communication at all levels of the teams, we created clinical, neurocognitive, and imaging workgroup workgroups, each of which is led by one member of the executive committee and includes at least one member from each site. Speaking once or twice per month, each workgroup is responsible for quality control in its domain. Specific quality control procedures are described below.

\section{Clinical assessments}

Prior to enrolling study subjects, reliability of the clinical raters across all sites for our diagnostic and clinical measures was confirmed, following best practices used in prior NIMH-funded clinical trials. First, we created a manual to standardize administration of core clinical measures across all sites. The manual contains general instructions for administration as well as guidelines for rating specific items on each measure. Second, each site provided typed transcripts of interviews completed with actual patients, and all raters scored them to ensure that interrater reliability was high across sites. All raters were required to be reliable on three versions of each core clinical measure prior to study start. Third, to ensure ongoing interrater reliability throughout the recruitment period, clinical raters will re-rate a random sampling of transcribed interviews from all sites each year and attend monthly conference calls to discuss and resolve any discrepant ratings. A second call each month will be used to discuss any other clinical issues that arise, including questions related to recruitment and eligibility.

\section{Neurocognitive testing}

We created a manual of standard operating procedures for the computerized neurocognitive protocol used in the study. Sites bought laptops with the same specifications (e.g., resolution, screen size, processor, random access memory (RAM), operating system) and standardized the set-up of the testing rooms to ensure that tasks would be presented consistently across sites. Each site tested the protocol with at least five volunteers to ensure proper use and output of the protocol, and a member of the team also reviewed screenshots and videos of each task at each site to confirm standard presentation of stimuli. Throughout study recruitment, output from each neurocognitive task will be reviewed periodically (e.g., after the first 10 subjects at each site are enrolled, then after the next 20 subjects) to ensure ongoing data quality. Members of the neurocognitive workgroup attend up to two conference calls each month to review administration of the tasks, discuss quality control, and ensure that data are being properly recorded and stored.

\section{Neuroimaging data}

At study start, we harmonized MRI data collection across the five sites so that raw MRI data (anatomical, DWI and rs-fMRI) can be optimally pooled. We followed established methods used in other multi-center MRI studies to reduce between-scanner effects [34, 134136]. These methods included: harmonization of scan sequence, brain coverage, and spatial resolution, as well as assessment of scan quality using two physical phantoms (i.e. National Institute of Standards and Technology (NIST) and Functional Biomedical Informatics Research Network ( $f B I R N)$ Agar). Two traveling humans were also scanned at all sites to assess within-subject scanner variability.

For continued quality assurance, physical phantoms will be scanned bi-monthly (NIST for geometry) and biweekly (fBIRN Agar for temporal stability). In addition, during the recruitment phase, all sites will send neuroimaging data of enrolled subjects to the Netherlands site within $48 \mathrm{~h}$ after a scan is acquired. The Netherlands site subsequently preprocesses incoming scans to check for correct spatial and timing parameters and to further ensure imaging quality during the recruitment phase by visual inspection and automated image quality measures of scanner or motion-related artefacts, scan coverage, and signal drop-out. Preprocessing and quality assurance is performed using open-source MRI processing suites such as FMRIB Software Library (FSL version 6.0.1, FMRIB, Oxford, UK), QUAD [137], and MRIQC [138]. Any anomalies are reported back to the site for follow-up. 


\section{Discussion}

Using harmonized methods for data collection and analysis, we will conduct the largest multimodal imaging and neurocognitive study in medication-free adults with OCD to date. Our study is designed to address a key question in the field: can we identify reproducible biosignatures of psychopathology that will change how we conceptualize mental illness, develop a mechanistic understanding of how current treatments work, and provide robust new targets for treatment development? As reviewed above, OCD provides an excellent test of this question, and the study described above represents the first step. Specifically, we will leverage our large diverse sample, multimodal imaging, and modern data-driven imaging methods [58, 133] to test whether we can empirically derive imaging signatures that reliably differentiate OCD patients from unaffected siblings and healthy controls.

With the increasing recognition of the global burden of mental illness $[67,139]$ and the fact that the International Classification of Diseases (ICD) will make OCD the lead disorder in a new cluster that includes OCD and related disorders [62], this is an opportune moment to focus the global health community on OCD by conducting an international study like this. Although there have been prior imaging and neurocognitive studies on OCD, ours will be the first to examine a very large sample of individuals who are free of medication and to use harmonized imaging methods and neurocognitive tasks in accordance with the RDoC domains. Our work will clarify whether OCD subjects across the globe show altered structure and function within frontal-striatal, frontal-limbic, and frontal-parietal circuits, as we hypothesize. If we see the structural abnormalities found in several meta- and mega-analyses [34, 35, $140,141]$, including that of ENIGMA-OCD [36, 37], it will provide strong evidence for the link between these structural abnormalities and OCD psychopathology. Moreover, we will test how multimodal imaging measures are linked to discrete domains of neurocognitive or clinical profiles, enabling us to make new discoveries about the association between abnormalities in structural and functional connectivity and cognitive and clinical dimensions of OCD.

Including unaffected siblings in our sample will contribute important new information about the brain circuit alterations associated with OCD risk and resilience, and we plan to recruit a sufficient sample of unaffected siblings to have the statistical power to examine these questions in a definitive way. Moreover, we will explore the relationship between environmental factors (childhood trauma, socioeconomic status, religiosity) and the brain signatures of OCD, as well as their potential moderating effect on the relationship between these signatures and OCD neurocognitive and clinical profiles. Consequently, this study will advance our understanding of the socio-contextual factors that influence OCD risk, severity, and associated morbidity, providing additional mechanisms to target for treatment and prevention.

Finally, our study is designed to strengthen the public health impact of research through large-scale global collaboration. By linking our five research sites, we will be able to recruit a large and diverse sample to examine questions that no single site alone could address. Moreover, by leveraging our intellectual resources and methodological expertise, we seek to accelerate discovery toward a circuit-based approach to cognitive and clinical dimensions of OCD. In the process, we integrate two different perspectives: that of global mental health, which has been focused on building capacity and service delivery in low-resource settings and closing the research and treatment gap, and that of translational neuroscience, which is focused on discovery of fundamental brain processes and mechanisms underlying psychopathology and using this knowledge to transform symptom-based approaches to diagnosis and treatment [142]). We integrate these perspectives by selecting imaging and neurocognitive measures that can be used in diverse settings (the "World Health Organization [WHO] approach") [66]; this way, our findings can be leveraged by many.

Empirically derived neural circuit taxonomies represent a new direction of discovery in psychiatry, and similar statistical methods have identified distinct biotypes in psychosis [143] as well as in depression and anxiety [144-147]. If we successfully link different brain circuit abnormalities to discrete cognitive and clinical profiles that are characteristic of OCD, we will be positioned for future study of how these brain circuit abnormalities develop during the course of disease, determine how they cut across traditional diagnostic boundaries, and use them as robust new treatment targets. Ultimately, this research could lead to objective methods for early diagnosis and intervention as well as to transdiagnostic treatments, with discoveries that will be relevant to populations across the globe.

\section{Abbreviations}

BABS: Brown Assessment of Beliefs Scale; CBT: Cognitive-behavioral therapy; CFI: Cultural Formulation Interview; CSTC: Cortico-striatal-thalamo-cortical: DWI: Diffusion weighted imaging; DY-BOCS: Dimensional Yale-Brown Obsessive-Compulsive Scale; ENIGMA: Enhancing Neuroimaging and Genetics through Meta-analyses; fBIRN: Functional Biomedical Informatics Research Network; HAM-A: Hamilton Anxiety Rating Scale; HAM-D: Hamilton Depression Rating Scale; HCs: Healthy control subjects; IQ: Intelligence quotient; MDD: Major depressive disorder; MRI: Magnetic resonance imaging; NIST: National Institute of Standards and Technology; OBIC: OCD Brain Imaging Consortium; PIs: Principal investigators; PSIR: Phase sensitive inversion recovery; RAM: Random access memory; RDoC: Research Domains Criteria; rs-fMRI: Resting state functional MRI; SCID: Structured Clinical Interview for DSM-5; SES: Socioeconomic status; USP-SPS: University of São Paulo Sensory Phenomena Scale; WAIS-IV: Wechsler Adult Intelligence Scale Fourth Edition; WAMI: Work and Meaning Inventory; WASI-I: Wechsler

Abbreviated Scale of Intelligence First Edition; WASI-II: Wechsler Abbreviated Scale of Intelligence Second Edition; WEIRD: Western, educated,

industrialized, rich, and democratic; WHODAS: World Health Organization 
Disability Assessment Schedule 2.0; YBOCS: Yale-Brown ObsessiveCompulsive Severity Scale

\section{Acknowledgements}

The authors would like to thanks Frances C. Robertson, Ph.D. and Petronella Samuels, B.Tech Radiography for their assistance with this project. Janardhan Reddy, Dan J. Stein, Roberto Lewis-Fernández and Melanie Wall are Principal Investigators on the grant from the National Institutes of Mental Health (R01

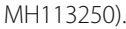

\section{Authors' contributions}

HBS, OAVDH, ECM, YCJR, DJS, RLF, MW, RGS, CL, PP, JCN, GV, and MQH contributed to the original conception of the study and writing the $\mathrm{NIMH}$ grant application as well as study set-up, data acquisition, and writing and/or substantially revising the draft of this manuscript. $\mathrm{DMH}, \mathrm{CV}$, and MCB contributed to drafting the manuscript, study set-up, and data acquisition. NB, AC, DLCC, NTDJ, MADM, FL, KM, CM, MN, MCGO, NP, BP, RR, KS, YRS, TVB, and SV contributed to the set-up of the study and/or data acquisition. MK and PVM contributed to data management. All authors read and approved the final manuscript.

\section{Funding}

Funding for this study is provided by a grant from the National Institutes of Mental Health (NIMH; 5R01MH113250-03) a grant agency where grants are competitively reviewed and funded, and supplemented at all sites by institutional resources and by philanthropic donations at the New York site. None of the funding sources played a role in designing the study.

\section{Availability of data and materials}

Not applicable.

\section{Ethics approval and consent to participate}

All sites completed human subjects training, received approval from their respective ethics boards, and will obtain written informed consent from all study subjects. The following ethics boards reviewed and approved the study: Brazil: National Committee of Ethics in Research, Ministry of Health, Federal Government of Brazil (reference number: 2.447.060); India: National Institute of Mental Health and Neuro Sciences; Netherlands: Medisch Ethische Toetsingscommissie at VU medisch centrum (VUmc; reference number 2017.296 - NL61982.029.17); South Africa: the Stellenbosch University's Health Research Ethics Committee (reference number N17/05/ 053) and the University of Cape Town's Human Research Ethics Committee (reference number: 358/2017); United States: the Institutional Review Board at the New York State Psychiatric Institute (reference number 7471).

\section{Consent for publication}

Not applicable.

\section{Competing interests}

In the past three years, HBS has received research support an industrysponsored clinical trial from Biohaven Pharmaceuticals, royalties from UpToDate Inc., and a stipend from the American Medical Association for her role as Associate Editor of JAMA Psychiatry. In the past 3 years, DJS has received research grants and/or consultancy honoraria from Lundbeck and Sun. DLCC has received consultancy honoraria from Pfizer and Libbs Pharmaceuticals, and a scholarship from Fundação de Amparo à Pesquisa do Estado de Sao Paulo (Sao Paulo State Foundation for Research Support). NMB is currently an editorial board member of the journal. The other others report no competing interests.

\section{Author details}

${ }^{1}$ Columbia University Irving Medical Center, Columbia University, New York, NY 10032, USA. ${ }^{2}$ The New York State Psychiatric Institute, New York, NY 10032, USA. ${ }^{3}$ Department of Psychiatry, Amsterdam UMC, Vrije Universiteit Amsterdam, de Boelelaan 1117, Amsterdam, Netherlands. ${ }^{4}$ Department of Anatomy and Neuroscience, Amsterdam UMC, Amsterdam Neuroscience, Vrije Universiteit Amsterdam, de Boelelaan 1117, Amsterdam, Netherlands. ${ }^{5}$ Obsessive-Compulsive Spectrum Disorders Program, Institute \& Department of Psychiatry, Hospital das Clinicas-HCFMUSP, University of Sao Paulo Medical School, Sao Paulo, Brazil. ${ }^{6}$ National Institute of Developmental Psychiatry, Sao Paulo, Brazil. ${ }^{7}$ National Institute of Mental Health \& Neurosciences
(NIMHANS), Bangalore, India. ${ }^{8}$ SAMRC Unit on Risk \& Resilience in Mental Disorders, Department of Psychiatry \& Neuroscience Institute, University of Cape Town, Cape Town, South Africa. ${ }^{9}$ SAMRC Unit on Risk \& Resilience in Mental Disorders, Department of Psychiatry, Stellenbosch University, Stellenbosch, South Africa. ${ }^{10}$ Department of Radiology and Nuclear Medicine, Amsterdam UMC, Vrije Universiteit Amsterdam, de Boelelaan 1117, Amsterdam, Netherlands. ${ }^{11}$ Amsterdam UMC, Vrije Universiteit, Psychiatry, Amsterdam Public Health Research Institute, de Boelelaan 1117, Amsterdam, Netherlands. ${ }^{12} \mathrm{GGZ}$ inGeest, Specialised Mental Health Care, Amsterdam, The Netherlands. ${ }^{13}$ Institute of Radiology, Hospital das Clinicas-HCFMUSP, University of Sao Paulo Medical School, Sao Paulo, Brazil.

Received: 2 December 2019 Accepted: 10 January 2020 Published online: 14 February 2020

\section{References}

1. Fontenelle LF, Mendlowicz MV, Versiani M. The descriptive epidemiology of obsessive-compulsive disorder. Prog Neuro-Psychopharmacol Biol Psychiatry. 2006;30(3):327-37.

2. Ruscio AM, Stein DJ, Chiu WT, Kessler RC. The epidemiology of obsessivecompulsive disorder in the National Comorbidity Survey Replication. Mol Psychiatry. 2010;15(1):53-63 2008/08/30.

3. Rosa AC, Diniz JB, Fossaluza V, Torres AR, Fontenelle LF, De Mathis AS, et al. Clinical correlates of social adjustment in patients with obsessivecompulsive disorder. J Psychiatr Res. 2012;46(10):1286-92.

4. Remmerswaal KCP, Batelaan NM, Smit JH, van Oppen P, van Balkom AJLM. Quality of life and relationship satisfaction of patients with obsessive compulsive disorder. J Obsessive Compuls Relat Disord. 2016;11:56-62.

5. Pérez-Vigil A, de la Cruz LF, Brander G, Isomura K, Jangmo A, Feldman I, et al. Association of Obsessive-Compulsive Disorder with Objective Indicators of educational attainment: a Nationwide register-based sibling control study. JAMA psychiatry. 2018;75(1):47-55.

6. de la Cruz LF, Rydell M, Runeson B, D'Onofrio BM, Brander G, Rück C, et al. Suicide in obsessive-compulsive disorder: a population-based study of 36 788 Swedish patients. Mol Psychiatry. 2017;22(11):1626.

7. Torres AR, Ramos-Cerqueira AT, Ferrão YA, Fontenelle LF, Miguel EC. Suicidality in obsessive-compulsive disorder: prevalence and relation to symptom dimensions and comorbid conditions. J Clin Psychiatry. 2011;72(1):17-26.

8. Eaton WW, Roth KB, Bruce M, Cottler L, Wu L, Nestadt G, et al. The relationship of mental and behavioral disorders to all-cause mortality in a 27-year follow-up of 4 epidemiologic catchment area samples. Am J Epidemiol. 2013;178(9):1366-77.

9. Meier SM, Mattheisen M, Mors O, Schendel DE, Mortensen PB, Plessen KJ. Mortality among persons with obsessive-compulsive disorder in Denmark. JAMA psychiatry. 2016;73(3):268-74

10. Demyttenaere K, Bruffaerts R, Posada-Villa J, Gasquet I, Kovess V, Lepine Jp, et al. Prevalence, severity, and unmet need for treatment of mental disorders in the World Health Organization World Mental Health Surveys. 2013;

11. Baxter AJ, Vos T, Scott KM, Ferrari AJ, Whiteford HA. The global burden of anxiety disorders in 2010. Psychol Med. 2014;44(11):2363-74.

12. Andlin-Sobocki $P$, Wittchen H. Cost of anxiety disorders in Europe. Eur J Neurol. 2005;12(s1):39-44.

13. Bloom DE, Cafiero E, Jané-Llopis E, Abrahams-Gessel S, Bloom LR, Fathima S, et al. The global economic burden of noncommunicable diseases. In: Program on the Global Demography of Aging; 2012.

14. Koran LM, Simpson HB. Guideline watch (March 2013): practice guideline for the treatment of patients with obsessive-compulsive disorder. Arlington: American Psychiatric Association; 2013.

15. Simpson HB, Foa EB, Liebowitz MR, Huppert JD, Cahill S, Maher MJ, et al. Cognitive-behavioral therapy vs risperidone for augmenting serotonin reuptake inhibitors in obsessive-compulsive disorder a randomized clinical trial. JAMA Psychiatry. 2013;70(11):1190-8.

16. Van Oppen P, Van Balkom AJLM, De Haan E, Van Dyck R. Cognitive therapy and exposure in vivo alone and in combination with fluvoxamine in obsessive-compulsive disorder: a 5-year follow-up. J Clin Psychiatry. 2005; 66(11):1415-22

17. Insel T, Cuthbert B, Garvey M, Heinssen R, Pine DS, Quinn K, et al. Research domain criteria (RDoC): toward a new classification framework for research on mental disorders. Am J Psychiatry. 2010;167(7):748-51.

18. International statistical classification of diseases and related health problems. 11th ed. Geneva: World Health Organization; 2018. 
19. Miguel EC, Do Rosário-Campos MC, Da Silva Prado H, Do Valle R, Rauch SL, Coffey BJ, et al. Sensory phenomena in obsessive-compulsive disorder and Tourette's disorder. J Clin Psychiatry. 2000;61:150-6.

20. Shavitt RG, de Mathis MA, Oki F, Ferrao YA, Fontenelle LF, Torres AR, Diniz $J B$, Costa DL, do Rosário MC, Hoexter MQ, Miguel EC. Phenomenology of OCD: lessons from a large multicenter study and implications for ICD-11. J psych res. 2014;57:141-8.

21. Ferrão YA, Shavitt RG, Prado H, Fontenelle LF, Malavazzi DM, de Mathis MA, et al. Sensory phenomena associated with repetitive behaviors in obsessivecompulsive disorder: an exploratory study of 1001 patients. Psychiatry Res. 2012;197(3):253-8.

22. Bloch MH, Landeros-Weisenberger A, Rosario MC, Pittenger C, Leckman JF. Meta-analysis of the symptom structure of obsessive-compulsive disorder. Am J Psychiatry. 2008;165(12):1532-42 2008/10/17.

23. Leckman JF, Denys D, Simpson HB, Mataix-Cols D, Hollander E, Saxena $\mathrm{S}$, et al. Obsessive-compulsive disorder: a review of the diagnostic criteria and possible subtypes and dimensional specifiers for DSM-V. Depress Anxiety. 2010;27(6):507-27 Available from: internal-pdf://111.210. 91.216/Insel_2010.pdf

24. du Mortier JAM, Visser HAD, van Balkom AJLM, van Megen HJGM, Hoogendoorn AW, Glas G, et al. Examining the factor structure of the selfreport Yale-Brown obsessive compulsive scale symptom checklist. Psychiatry Res. 2019;271:299-305.

25. Milad MR, Rauch SL. Obsessive-compulsive disorder: beyond segregated cortico-striatal pathways. Trends Cogn Sci. 2012;16(1):43-51 Available from: http://www.ncbi.nlm.nih.gov/pubmed/22138231. 2011/12/06

26. van den Heuvel OA, van Wingen G, Soriano-Mas C, Alonso P, Chamberlain SR, Nakamae T, et al. Brain circuitry of compulsivity. Eur Neuropsychopharmacol. 2016;26(5):810-27.

27. Henrich J, Heine SJ, Norenzayan A. The weirdest people in the world? Behav Brain Sci. 2010;33(2-3):61-83

28. Pauls DL, Abramovitch A, Rauch SL, Geller DA. Obsessive-compulsive disorder: An integrative genetic and neurobiological perspective. Nat Rev Neurosci. 2014;15:410-24.

29. Gottlich M, Kramer UM, Kordon A, Hohagen F, Zurowski B. Decreased limbic and increased fronto-parietal connectivity in unmedicated patients with obsessive-compulsive disorder. Hum Brain Mapp. 2014;35(11):5617-32 2014/ $07 / 22$

30. Hartmann T, Vandborg S, Rosenberg R, Sorensen L, Videbech P. Increased fractional anisotropy in cerebellum in obsessive-compulsive disorder. Acta Neuropsychiatr. 2015/11/03;2015:1-8.

31. Subira M, Cano M, de Wit SJ, Alonso P, Cardoner N, Hoexter MQ, et al. Structural covariance of neostriatal and limbic regions in patients with obsessive-compulsive disorder. J Psychiatry Neurosci. 2015;41(1):150012 2015/10/28

32. Narayanaswamy JC, Jose D, Kalmady SV, Agarwal SM, Venkatasubramanian G, Reddy YJ. Cerebellar volume deficits in medication-naïve obsessive compulsive disorder. Psychiatry Res Neuroimaging. 2016;254:164-8.

33. Boedhoe P, Van den Heuvel OA. The structure of the OCD brain. In: Pittenger C, editor. Obsessive-compulsive Disorder Phenomenology, Pathophysiology, and Treatment. New York: Oxford University Press; 2017.

34. de Wit SJ, Alonso P, Schweren L, Mataix-Cols D, Lochner C, Menchón JM, et al. Multicenter voxel-based morphometry mega-analysis of structural brain scans in obsessive-compulsive disorder. Am J Psychiatry. 2014;171(3):340-9.

35. Boedhoe PS, van Rooij D, Hoogman M, Thompson PM, Stein DJ, Buitelaar JK, Franke B, van den Heuvel OA. ENIGMA-ADHD working group, ENIGMAASD working group, ENIGMA-OCD working group. Subcortical brain volume, regional cortical thickness and cortical surface area across attention-deficit/hyperactivity disorder (ADHD), autism spectrum disorder (ASD), and obsessive-compulsive disorder (OCD): findings from the ENIGMAADHD,-ASD, and-OCD working groups. bioRxiv. 2019:673012.

36. Boedhoe PS, Schmaal L, Abe Y, Ameis SH, Arnold PD, Batistuzzo MC, et al. Distinct subcortical volume alterations in pediatric and adult OCD: a worldwide meta- and mega-analysis. Am J Psychiatry. 2017;174(1):60-9.

37. Boedhoe PS, Schmaal L, Abe Y, Alonso P, Ameis SH, Anticevic A, et al. Cortical abnormalities associated with pediatric and adult obsessivecompulsive disorder: findings from the ENIGMA obsessive-compulsive disorder working group. Am J Psychiatry. 2018;175(5):453-62.

38. Koch K, Reess TJ, Rus OG, Zimmer C, Zaudig M. Diffusion tensor imaging (DTI) studies in patients with obsessive-compulsive disorder (OCD): a review. J Psychiatr Res. 2014;54:26-35 2014/04/04.
39. Radua J, Grau M, van den Heuvel OA, de Schotten MT, Stein DJ, CanalesRodríguez EJ, et al. Multimodal voxel-based Meta-analysis of white matter abnormalities in obsessive-compulsive disorder. Neuropsychopharmacology. 2014;39(7):1547-57.

40. Rosso IM, Olson EA, Britton JC, Stewart SE, Papadimitriou G, Killgore WD, et al. Brain white matter integrity and association with age at onset in pediatric obsessive-compulsive disorder. Biol Mood Anxiety Disord. 2014; 4(1):13 2014/12/30.

41. Fan S, van den Heuvel OA, Cath DC, van der Werf YD, de Wit SJ, de Vries FE, et al. Mild white matter changes in un-medicated obsessive-compulsive disorder patients and their unaffected siblings. Front Neurosci. 2016:9:495.

42. Glahn A, Prell T, Grosskreutz J, Peschel T, Muller-Vahl KR. Obsessivecompulsive disorder is a heterogeneous disorder: evidence from diffusion tensor imaging and magnetization transfer imaging. BMC Psychiatry. 2015; 15:135 2015/06/26.

43. Magioncalda P, Martino M, Ely BA, Inglese M, Stern ER. Microstructural white-matter abnormalities and their relationship with cognitive dysfunction in obsessive-compulsive disorder. Brain Behav. 2016;6(3):e00442 2016/04/22.

44. Harrison BJ, Soriano-Mas C, Pujol J, Ortiz H, Lopez-Sola M, Hernandez-Ribas $\mathrm{R}$, et al. Altered corticostriatal functional connectivity in obsessivecompulsive disorder. Arch Gen Psychiatry. 2009;66(11):1189-200.

45. Stern ER, Fitzgerald KD, Welsh RC, Abelson JL, Taylor SF. Resting-state functional connectivity between fronto-parietal and default mode networks in obsessive-compulsive disorder. PLoS One. 2012;7(5):e36356 2012/05/10.

46. Harrison BJ, Pujol J, Cardoner N, Deus J, Alonso P, Lopez-Sola M, et al. Brain corticostriatal systems and the major clinical symptom dimensions of obsessive-compulsive disorder. Biol Psychiatry. 2013;73(4):321-8 Available from: http://www.ncbi.nlm.nih.gov/pubmed/23200527.

47. Posner J, Marsh R, Maia TV, Peterson BS, Gruber A, Simpson HB. Reduced functional connectivity within the limbic cortico-striato-thalamo-cortical loop in unmedicated adults with obsessive-compulsive disorder. Hum Brain Mapp. 2014;35(6):2852-60 2013/10/15

48. Beucke JC, Sepulcre J, Eldaief MC, Sebold M, Kathmann N, Kaufmann C Default mode network subsystem alterations in obsessive-compulsive disorder. Br J Psychiatry. 2014:205(5):376-82.

49. Gruner $P$, Vo A, Argyelan M, Ikuta T, Degnan AJ, John M, Peters BD, Malhotra AK, Uluğ AM, Szeszko PR. Independent component analysis of resting state activity in pediatric obsessive-compulsive disorder. Human brain mapping. 2014;35(10):5306-15

50. Yang X, Hu X, Tang W, Li B, Yang Y, Gong Q, et al. Intrinsic brain abnormalities in drug-naive patients with obsessive-compulsive disorder: a resting-state functional MRI study. J Affect Disord. 2019;245:861-8.

51. Gürsel DA, Avram M, Sorg C, Brandl F, Koch K. Frontoparietal areas link impairments of large-scale intrinsic brain networks with aberrant frontostriatal interactions in OCD: a meta-analysis of resting-state functional connectivity. Neurosci Biobehav Rev. 2018;87:151-60.

52. Yang $X$, Luo J, Zhong Z, Yang $X$, Yao S, Wang $P$, et al. Abnormal regional homogeneity in patients with obsessive-compulsive disorder and their unaffected siblings: a resting-state fMRI study. Front Psychiatry. 2019;10:452.

53. Xu T, Zhao Q, Wang P, Fan Q, Chen J, Zhang $H$, et al. Altered resting-state cerebellar-cerebral functional connectivity in obsessive-compulsive disorder. Psychol Med. 2019;49(7):1156-65.

54. de Vries FE, de Wit SJ, van den Heuvel OA, Veltman DJ, Cath DC, van Balkom AJLM, et al. Cognitive control networks in OCD: a resting-state connectivity study in unmedicated patients with obsessive-compulsive disorder and their unaffected relatives. World J Biol Psychiatry. 2019;20(3):230-42.

55. Fan Q, Yan X, Wang J, Chen Y, Wang X, Li C, et al. Abnormalities of white matter microstructure in unmedicated obsessive-compulsive disorder and changes after medication. PLoS One, 2012/05/05. 2012; 7(4):e35889

56. Benedetti F, Giacosa C, Radaelli D, Poletti S, Pozzi E, Dallaspezia S, et al. Widespread changes of white matter microstructure in obsessivecompulsive disorder: effect of drug status. Eur Neuropsychopharmacol. 2013;23(7):581-93 2012/09/08.

57. Posner J, Hellerstein DJ, Gat I, Mechling A, Klahr K, Wang Z, et al. Antidepressants normalize the default mode network in patients with dysthymia. JAMA Psychiatry. 2013:70(4):373-82 2013/02/08.

58. Calhoun VD, Sui J. Multimodal fusion of brain imaging data: A key to finding the missing link(s) in complex mental illness. Biol Psychiatry Cogn Neurosci Neuroimaging. 2016;3(1):230-44 2016/06/28. 
59. Chen Y, Juhas M, Greenshaw AJ, Hu Q, Meng X, Cui H, et al. Abnormal resting-state functional connectivity of the left caudate nucleus in obsessive-compulsive disorder. Neurosci Lett. 2016;623:57-62 2016/05/05.

60. Stein DJ, Costa DL, Lochner C, Miguel EC, Reddy YCJ, Shavitt RG, et al. Obsessive-compulsive disorder. Nat Rev Dis Prim. 2019;5(1):1-21.

61. American Psychiatric Association. Diagnostic and statistical manual of mental disorders (DSM-5 ${ }^{\circledast}$ ). Arlington: American Psychiatric Pub; 2013. p. 991.

62. Stein DJ, Kogan CS, Atmaca M, Fineberg NA, Fontenelle LF, Grant JE, et al. The classification of Obsessive-Compulsive and Related Disorders in the ICD-11. J Affect Disord. 2015;190:663-74 2015/11/23.

63. Hofmeijer-Sevink MK, Van Oppen P, Van Megen HJ, Batelaan NM, Cath DC, Van Der Wee NJA, et al. Clinical relevance of comorbidity in obsessive compulsive disorder: the Netherlands OCD association study. J Affect Disord. 2013;150(3):847-54.

64. Visser H, van Megen H, van Oppen P, Hoogendoorn A, Glas G, Neziroglu F, et al. The impact of poor insight on the course of obsessive-compulsive disorder in patients receiving naturalistic treatment. J Obsessive Compuls Relat Disord. 2017;13:42-8.

65. van den Heuvel OA, Remijnse PL, Mataix-Cols D, Vrenken H, Groenewegen $\mathrm{HJ}$, Uylings HB, et al. The major symptom dimensions of obsessivecompulsive disorder are mediated by partially distinct neural systems. Brain. 2008;132(4):853-68.

66. Reed GM, Ayuso-Mateos JL. Towards a more clinically useful International World Health Organisation classification of mental disorders. Rev Psiquiatr Salud Ment. 2011;4(3):113-6 2011/07/01.

67. Insel TR, Collins PY, Hyman SE. Darkness invisible. Foreign Aff. 2015;94(1):189.

68. Simpson HB, Kegeles LS, Hunter L, Mao X, Van Meter P, Xu X, et al. Assessment of glutamate in striatal subregions in obsessive-compulsive disorder with proton magnetic resonance spectroscopy. Psychiatry Res. 2015;232(1):65-70 2015/02/27.

69. No Title [Internet]. Available from: http://enigma.ini.usc.edu/ongoing/ enigma-ocd-working-group/.

70. de Wit SJ, de Vries FE, van der Werf YD, Cath DC, Heslenfeld DJ, Veltman EM, et al. Presupplementary motor area hyperactivity during response inhibition: a candidate endophenotype of obsessive-compulsive disorder. Am J Psychiatry. 2012;169(10):1100-8.

71. de Vries FE, de Wit SJ, Cath DC, van der Werf YD, van der Borden V, van Rossum TB, et al. Compensatory frontoparietal activity during working memory: an endophenotype of obsessive-compulsive disorder. Biol Psychiatry. 2014;76(11):878-87.

72. Menzies L, Achard S, Chamberlain SR, Fineberg N, Chen CH, Del Campo N, et al. Neurocognitive endophenotypes of obsessive-compulsive disorder. Brain. 2007;130(12):3223-36.

73. Shaw P, Sharp W, Sudre G, Wharton A, Greenstein D, Raznahan A, et al. Subcortical and cortical morphological anomalies as an endophenotype in obsessive-compulsive disorder. Mol Psychiatry. 2015;20(2):224-31.

74. Thorsen AL, de Wit SJ, de Vries FE, Cath DC, Veltman DJ, van der Werf $Y D$, et al. The endophenotype of emotional regulation in obsessivecompulsive disorder (OCD). Eur Neuropsychopharmacol. 2017;27(6):619.

75. Fontenelle LF, Mendlowicz MV, Marques C, Versiani M. Trans-cultural aspects of obsessive-compulsive disorder: a description of a Brazilian sample and a systematic review of international clinical studies. J Psychiatr Res. 2004;38(4):403-11.

76. Lewis-Fernandez R, Hinton DE, Laria AJ, Patterson EH, Hofmann SG, Craske $M G$, et al. Culture and the anxiety disorders: recommendations for DSM-V. Depress Anxiety. 2010;27(2):212-29 Available from: http://www.ncbi.nlm.nih. gov/entrez/query.fcgi?cmd=Retrieve\&db=PubMed\&dopt=Citation\&list_uids= 20037918. 2009/12/29

77. Matsunaga H, Seedat S. Obsessive-compulsive spectrum disorders: crossnational and ethnic issues. CNS Spectr. 2007;12(5):392-400.

78. Stein DJ, Rapoport JL. Cross-cultural studies and obsessive-compulsive disorder. CNS Spectr. 1996;1(1):42-6.

79. Adams TG, Kelmendi B, Brake CA, Gruner P, Badour CL, Pittenger C. The role of stress in the pathogenesis and maintenance of obsessive-compulsive disorder. Chronic Stress. 2018;2:247054701875804.

80. Miller ML, Brock RL. The effect of trauma on the severity of obsessive-compulsive spectrum symptoms: A meta-analysis. J Anxiety Disord. 2017;47:29-44.

81. Grisham JR, Fullana MA, Mataix-Cols D, Moffitt TE, Caspi A, Poulton R. Risk factors prospectively associated with adult obsessive-compulsive symptom dimensions and obsessive-compulsive disorder. Psychol Med. 2011;41(12): 2495-506.
82. Holz NE, Boecker R, Hohm E, Zohsel K, Buchmann AF, Blomeyer D, et al. The Long-Term Impact of Early Life Poverty on Orbitofrontal Cortex Volume in Adulthood: Results from a Prospective Study Over 25 Years. Neuropsychopharmacology. 2014; Available from: internal-pdf://116.127.32. 48/Insel_2010.pdf.

83. Ansell EB, Rando K, Tuit K, Guarnaccia J, Sinha R. Cumulative adversity and smaller gray matter volume in medial prefrontal, anterior cingulate, and insula regions. Biol Psychiatry. 2012;72(1):57-64.

84. Sripada RK, Swain JE, Evans GW, Welsh RC, Liberzon I. Childhood poverty and stress reactivity are associated with aberrant functional connectivity in default mode network. Neuropsychopharmacology. 2014;39(9):2244.

85. Weinberger DR, Radulescu E. Finding the Elusive Psychiatric "Lesion" With 21st-Century Neuroanatomy: A Note of Caution. Am J Psychiatry. 2016; 173(1):27-33 2015/09/01

86. McLaughlin KA, Sheridan MA, Lambert HK. Childhood adversity and neural development: deprivation and threat as distinct dimensions of early experience. Neurosci Biobehav Rev. 2014;47:578-91 2014/12/03.

87. Nemeroff CB, Binder E. The preeminent role of childhood abuse and neglect in vulnerability to major psychiatric disorders: toward elucidating the underlying neurobiological mechanisms. J Am Acad Child Adolesc Psychiatry. 2014;53(4):395-7 2014/03/25.

88. Teicher MH, Samson JA. Childhood maltreatment and psychopathology: A case for ecophenotypic variants as clinically and neurobiologically distinct subtypes. Am J Psychiatry. 2013;170(10):1114-33 2013/08/29.

89. Marusak HA, Martin KR, Etkin A, Thomason ME. Childhood trauma exposure disrupts the automatic regulation of emotional processing. Neuropsychopharmacology. 2015;40(5):1250-8 2014/11/22.

90. De Bellis MD, Zisk A. The biological effects of childhood trauma. Child Adolesc Psychiatr Clin N Am. 2014;23(2):185-222 vii. 2014/03/25.

91. Brooks SJ, Naidoo V, Roos A, Fouche JP, Lochner C, Stein DJ. Early-life adversity and orbitofrontal and cerebellar volumes in adults with obsessivecompulsive disorder: voxel-based morphometry study. The British Journal of Psychiatry. 2016;208(1):34-41.

92. Lewis-Fernandez R, Aggarwal NK, Hinton L, Hinton DE, Kirmayer LJ, editors. DSM- $5^{\oplus}$ handbook on the cultural formulation interview. Washington, DC: American Psychiatric Association Publishing; 2016.

93. Goodman WK. The Yale-Brown Obsessive Compulsive Scale: I. Development, use, and reliability. Arch Gen Psychiatry. 1989;46(11):1006. Available from. https://doi.org/10.1001/archpsyc.1989.01810110048007.

94. Goodman WK, Price LH, Rasmussen SA, Mazure C, Delgado P, Heninger GR, Charney DS. The yale-brown obsessive compulsive scale: II. Validity. Archives of general psychiatry. 1989;46(11):1012-6.

95. Wechsler D. WASI manual. Wechsler abbreviated scale of intelligence. San Antonio, TX: The Psychological Corporation; 1999.

96. Kamat V. Measuring intelligence of Indian children. 4th ed. London: Oxford University Press; 1964.

97. Wechsler D. Wechsler adult intelligence scale -- fourth edition. San Antonio: Pearson; 2008.

98. Wechsler D, Hsiao-pin C. WASI-II Wechsler Abbreviated Scale Intell. San Antonio: NCS Pearson; 2011.

99. Foa EB, Huppert JD, Leiberg S, Langner R, Kichic R, Hajcak G, et al. The obsessive-compulsive inventory: development and validation of a short version. Psychol Assess. 2002;14(4):485.

100. Rosario-Campos MC, Miguel EC, Quatrano S, Chacon P, Ferrao Y, Findley D, et al. The Dimensional Yale-Brown Obsessive-Compulsive Scale (DY-BOCS): an instrument for assessing obsessive-compulsive symptom dimensions. Mol Psychiatry. 2006;11(5):495-504.

101. Eisen JL. The Brown assessment of beliefs scale: reliability and validity. Am J Psychiatry. 1998;155(1):102-8.

102. Rosario MC, Prado HS, Borcato S, Diniz JB, Shavitt RG, Hounie AG, et al. Validation of the University of São Paulo sensory phenomena scale: initial psychometric properties. CNS Spectr. 2009;14(6):315-23.

103. First MB, Williams JB, Karg RS, Spitzer RL. Structured Clinical Interview for DSM-5-Research Version (SCID-5 for DSM-5, Research Version; SCID-5-RV). Arlington: American Psychiatric Association; 2015.

104. Hamilton M. A rating scale for depression. Psychiatry: J Neurol Meurosurgery; 1960.

105. Hamilton MC, Schutte NS, Malouff JM. Hamilton anxiety scale (HAMA) Sourcebook of Adult Assessment: Applied Clinical Psychology. 1976:154-7.

106. Baron-Cohen S, Wheelwright S, Skinner R, Martin J, Clubley E. The autismspectrum quotient $(A Q)$ : evidence from asperger syndrome/high- 
functioning autism, malesand females, scientists and mathematicians. J Autism Dev Disord. 2001;31(1):5-17.

107. Van Overveld WJM, de Jong PD, Peters ML, Cavanagh K, Davey GCL. Disgust propensity and disgust sensitivity: separate constructs that are differentially related to specific fears. Pers Individ Differ. 2006;41(7):1241-52.

108. Guo K, Youssef GJ, Dawson A, Parkes L, Oostermeijer S, López-Solà C, et al. A psychometric validation study of the Impulsive-Compulsive Behaviours Checklist: A transdiagnostic tool for addictive and compulsive behaviours. Addict Behav. 2017;67:26-33.

109. Üstün TB. Measuring health and disability: manual for WHO disability assessment schedule WHODAS 2.0: World Health Organization; 2010.

110. Steger MF, Dik BJ, Duffy RD. Measuring meaningful work: the work and meaning inventory (WAMI). J Career Assess. 2012;20(3):322-37.

111. Bernstein DP, Stein JA, Newcomb MD, Walker E, Pogge D, Ahluvalia T, et al. Development and validation of a brief screening version of the childhood trauma questionnaire. Child Abuse Negl. 2003;27(2):169-90.

112. Inozu M, Ulukut FO, Ergun G, Alcolado GM. The mediating role of disgust sensitivity and thought-action fusion between religiosity and obsessive compulsive symptoms. Int J Psychol. 2014;49(5):334-41.

113. No Title [Internet]. Available from: https://www.nimh.nih.gov/research/ research-funded-by-nimh/rdoc/constructs/rdoc-matrix.shtml.

114. van den Heuvel OA, Groenewegen HJ, Barkhof F, Lazeron RHC, van Dyck R, Veltman DJ. Frontostriatal system in planning complexity: a parametric functional magnetic resonance version of tower of London task. Neuroimage. 2003;18(2):367-74.

115. van den Heuvel OA, Veltman DJ, Groenewegen HJ, Witter MP, Merkelbach J, Cath DC, et al. Disorder-specific neuroanatomical correlates of attentional bias in obsessive-compulsive disorder, panic disorder, and hypochondriasis. Arch Gen Psychiatry. 2005;62(8):922-33 Available from: http://www.ncbi.nlm. nih.gov/entrez/query.fcgi?cmd=Retrieve\&db=PubMed\&dopt=Citation\&list_ uids=16061770. 2005/08/03.

116. Bari A, Robbins TW. Inhibition and impulsivity: behavioral and neural basis of response control. Prog Neurobiol. 2013;108:44-79.

117. van Velzen LS, Vriend C, de Wit SJ, van den Heuvel OA. Response inhibition and interference control in obsessive-compulsive spectrum disorders. Front Hum Neurosci. 2014;8:419.

118. Kable JW, Glimcher PW. The neural correlates of subjective value during intertemporal choice. Nat Neurosci. 2007;10(12):1625-33.

119. Figner B, Knoch D, Johnson EJ, Krosch AR, Lisanby SH, Fehr E, et al. Lateral prefrontal cortex and self-control in intertemporal choice. Nat Neurosci. 2010;13(5):538-9.

120. Figee $M$, Vink $M$, de Geus F, Vulink N, Veltman DJ, Westenberg $H$, et al. Dysfunctional reward circuitry in obsessive-compulsive disorder. Biol Psychiatry. 2011;69(9):867-74

121. Steinglass JE, Lempert KM, Choo TH, Kimeldorf MB, Wall M, Walsh BT, et al. Temporal discounting across three psychiatric disorders: anorexia nervosa, obsessive compulsive disorder, and social anxiety disorder. Depress Anxiety. 2017;34(5):463-70.

122. Milad MR, Furtak SC, Greenberg JL, Keshaviah A, Im JJ, Falkenstein MJ, et al. Deficits in conditioned fear extinction in obsessive-compulsive disorder and neurobiological changes in the fear circuit. JAMA psychiatry. 2013;70(6):608-18.

123. Kami A, Meyer G, Jezzard P, Adams MM, Turner R, Ungerleider LG. Functional MRI evidence for adult motor cortex plasticity during motor skill learning. Nature. 1995;377(6545):155.

124. Witt ST, Laird AR, Meyerand ME. Functional neuroimaging correlates of finger-tapping task variations: an ALE meta-analysis. Neuroimage. 2008;42(1): 343-56.

125. Da Silva FN, Irani F, Richard J, Brensinger CM, Bilker WB, Gur RE, et al. More than just tapping: index finger-tapping measures procedural learning in schizophrenia. Schizophr Res. 2012;137(1-3):234-40 2012/02/22.

126. Vahdat S, Fogel S, Benali H, Doyon J. Network-wide reorganization of procedural memory during NREM sleep revealed by fMRI. Elife. 2017;6: e24987.

127. Daw ND, Gershman SJ, Seymour B, Dayan P, Dolan RJ. Model-based influences on humans' choices and striatal prediction errors. Neuron. 2011; 69(6):1204-15

128. Decker JH, Otto AR, Daw ND, Hartley CA. From creatures of habit to goaldirected learners: tracking the developmental emergence of model-based reinforcement learning. Psychol Sci. 2016;27(6):848-58.
129. Simpson HB, Rosen W, Huppert JD, Lin SH, Foa EB, Liebowitz MR. Are there reliable neuropsychological deficits in obsessive-compulsive disorder? J Psychiatr Res. 2006;40(3):247-57.

130. Abramovitch A, Abramowitz JS, Mittelman A. The neuropsychology of adult obsessive-compulsive disorder: a meta-analysis. Clin Psychol Rev. 2013;33(8): $1163-71$.

131. Snyder HR, Kaiser RH, Warren SL, Heller W. Obsessive-compulsive disorder is associated with broad impairments in executive function: A meta-analysis. Clin Psychol Sci. 2015;3(2):301-30 2015/03/11

132. Abramovitch A, Anholt G, Raveh-Gottfried S, Hamo N, Abramowitz JS. MetaAnalysis of Intelligence Quotient $(\mathrm{IQ})$ in Obsessive-Compulsive Disorder. Neuropsychol Rev. 2018;28:111-20.

133. De Domenico M. Multilayer modeling and analysis of human brain networks. GigaScience. 2017:6:1-8.

134. Jack CR Jr, Bernstein MA, Fox NC, Thompson P, Alexander G, Harvey D, et al. The Alzheimer's Disease Neuroimaging Initiative (ADNI): MRI methods. J Magn Reson Imaging. 2008;27(4):685-91 2008/02/28.

135. Poldrack RA, Gorgolewski KJ. Making big data open: data sharing in neuroimaging. Nat Neurosci. 2014;17(11):1510-7.

136. Glover GH, Mueller BA, Turner JA, van Erp TG, Liu TT, Greve DN, et al. Function biomedical informatics research network recommendations for prospective multicenter functional MRI studies. J Magn Reson Imaging. 2012;36(1):39-54 Available from: http://search.ebscohost.com/login. aspx?direct=true\&AuthType=cookie,ip,shib,uid\&db=rzh\&AN=104462801 \&site=ehost-live\&scope=site\&authtype=shib\&custid=s8000044.

137. Bastiani M, Cottaar M, Fitzgibbon SP, Suri S, Alfaro-Almagro F, Sotiropoulos SN, et al. Automated quality control for within and between studies diffusion MRI data using a non-parametric framework for movement and distortion correction. Neuroimage. 2019;184:801-12.

138. Esteban O, Poldrack RA, Gorgolewski KJ. Improving out-of-sample prediction of quality of MRIQC. In: Lecture Notes in Computer Science (including subseries Lecture Notes in Artificial Intelligence and Lecture Notes in Bioinformatics); 2018. p. 190-9.

139. Stein D. Obsessive-compulsive disorder and global mental health. Indian J Psychiatry. 2019;61(7):4.

140. Radua J, Mataix-Cols D. Voxel-wise meta-analysis of grey matter changes in obsessive-compulsive disorder. Br J Psychiatry. 2009;195(5):393-402.

141. Radua J, van den Heuvel OA, Surguladze S, Mataix-Cols D. Meta-analytical comparison of voxel-based morphometry studies in obsessive-compulsive disorder vs other anxiety disorders. Arch Gen Psychiatry. 2010;67(7):701-11.

142. Stein DJ, He Y, Phillips A, Sahakian BJ, Williams J, Patel V. Global mental health and neuroscience: potential synergies. Lancet Psychiatry. 2015;2(2): 178-85.

143. Clementz BA, Sweeney JA, Hamm JP, Ivleva El, Ethridge LE, Pearlson GD, et al. Identification of Distinct Psychosis Biotypes Using Brain-Based Biomarkers. Am J Psychiatry. 2016;173(4):373-84 2015/12/15.

144. Williams LM. Precision psychiatry: a neural circuit taxonomy for depression and anxiety. Lancet Psychiatry. 2016;3(5):472-80 2016/05/08.

145. Drysdale AT, Grosenick L, Downar J, Dunlop K, Mansouri F, Meng Y, et al. Resting-state connectivity biomarkers define neurophysiological subtypes of depression. Nat Med. 2017;23(1):28-38.

146. Watters AJ, Korgaonkar MS, Carpenter JS, Harris AW, Gross JJ, Williams LM. Profiling risk for depressive disorder by circuit, behavior and self-report measures of emotion function. J Affect Disord. 2017;227:595-602.

147. Williams LM. Defining biotypes for depression and anxiety based on largescale circuit dysfunction: a theoretical review of the evidence and future directions for clinical translation. Depress Anxiety. 2017;34(1):9-24.

\section{Publisher's Note}

Springer Nature remains neutral with regard to jurisdictional claims in published maps and institutional affiliations. 